\title{
SISTEMA REPRODUTIVO DE OXYPETALUM APPENDICULATUM MART. E OXYPETALUM BANKSII ROEM. \& SCHULT. SUBSP. BANKSII (ASCLEPIADACEAE) ${ }^{1}$
}

\author{
Milene Faria Vieira ${ }^{2}$ \\ George John Shepherd ${ }^{3}$
}

\section{Recebido em 28/01/1998. Aceito em 27/07/1999}

\begin{abstract}
RESUMO - (Sistema reprodutivo de Oxypetalum appendiculatum Mart. e Oxypetalum banksii Roem. \& Schult. subsp. banksii (Asclepiadaceae)). Estudos sobre o sistema reprodutivo de $O$. appendiculatum e $O$. banksii subsp. banksii foram realizados em Viçosa, MG, sudeste do Brasil. Ambas são autocompatíveis e a viabilidade das sementes, resultantes de autopolinizações e polinizações cruzadas, é alta. A baixa taxa de produção de frutos em flores de $O$. banksii subsp. banksii expostas aos visitantes (polinizações abertas) deve estar relacionada à falta de polinização (fator extrínseco). Em $O$. appendiculatum, a baixa taxa de produção de frutos deve estar relacionada a fatores intrínsecos, regulados pela planta. Polinizações interespecíficas resultaram em sementes viáveis e os híbridos $F_{1}$ apresentaram $64 \%$ de flores férteis; esses híbridos não foram encontrados na natureza. Autopolinizações em flores de $F_{1}$ resultaram em sementes também viáveis.
\end{abstract}

Palavras-chave - Asclepiadaceae, compatibilidade, polinização interespecífica, Oxypetalum

\begin{abstract}
Breeding systems in Oxypetalum appendiculatum Mart. and Oxypetalum banksii Roem. \& Schult. subsp. banksii (Asclepiadaceae)). Studies on the breeding systems of $O$. appendiculatum and $O$. banksii subsp. banksi were carried out at Viçosa, Minas Gerais State, southeastern Brazil. Both species are self-compatible and seeds that resulted from self- and cross-pollinations presented a high viability. The low rate of fruit set in $O$. banksii subsp. banksii flowers exposed to visitors (open pollination) may be related to the lack of pollination (extrinsic factor). In $O$. appendiculatum the low rate of fruit set can be related to intrinsic factors, regulated by the plant itself. Interspecific pollinations resulted in viable seeds and the $F_{1}$ hybrid presented $64 \%$ of fertile flowers; this hybrid has not yet been found under natural conditions. Self-pollinations in $\mathrm{F}_{\mathrm{I}}$ flowers also yielded viable seeds.
\end{abstract}

Key words - Asclepiadaceae, compatibility, interspecific pollination, Oxypetalum

\section{Introdução}

Asclepiadaceae possui cerca de 300 gêneros e 2.200 espécies distribuídas principalmente nas regiões tropicais e subtropicais do mundo (Rosatti 1989; Swarupanandan et al. 1996). No Brasil, foram registrados cerca de 45 gêneros (Barroso et al. 1986), entre eles Oxypetalum R. Br., restrito à região neotropical (Mabberley 1997).

Há alguma informação sobre a biologia reprodutiva de Asclepiadaceae em apenas $6 \%$ das espécies (Ollerton \& Liede 1996). Os estudos

\footnotetext{
1 Parte da Tese de Doutorado da primeira Autora; trabalho apresentado no 49 "Congresso Nacional de Botânica, Salvador, BA

2 Departamento de Biologia Vegetal, Universidade Federal de Viçosa, CEP 36.571-000, Viçosa, MG, Brasil e-mail: mfvieira@mail.ufv.br

3 Departamento de Botânica, IB, UNICAMP, C. Postal 6109, CEP 13.083-970, Campinas, SP, Brasil
} 
sobre o sistema reprodutivo são restritos às espécies de Asclepias (Sparrow \& Pearson 1948; Kephart 1981; Wyatt \& Broyles 1994; 1997). Essas plantas apresentam, segundo Wyatt \& Broyles (1994), duas categorias de sistemas de compatibilidade: aquelas que são principal ou inteiramente auto-incompatíveis e aquelas que são principal ou inteiramente autocompatíveis. Em populações naturais, a taxa de frutificação dessas plantas é baixa, geralmente de 1 a $5 \%$ (Wyatt \& Broyles 1990; 1994).

Há vários registros de híbridos naturais e artificiais entre espécies de Asclepias (Stevens 1945; Woodson 1954; Kephart et al. 1988). Wyatt \& Hunt (1991) comentaram que espécies simpátricas desse gênero ocasionalmente se intercruzam e que retrocruzamentos e, conseqüentemente, introgressões devem ocorrer.

$\mathrm{O}$ objetivo do presente estudo foi analisar o sistema reprodutivo de Oxypetalum appendiculatum e $O$. banksii subsp. banksii, inclusive a compatibilidade entre esses táxons.

\section{Material e métodos}

O. appendiculatum e $O$. banksii subsp. banksii são trepadeiras, ocupam áreas similares ou sobrepostas, com flores que duram em média cerca de oito dias e apresentam sobreposição no período de floração, além de possuírem insetos polinizadores em comum (Vieira 1998). $O$. appendiculatum é encontrada em cerradão, cerrado, campo limpo, rupestre e de altitude, mata ciliar, áreas brejosas, pastos e beiras de estradas; ocorre nos Estados do sul e sudeste brasileiro, no Paraguai e na Argentina (Occhioni 1953; FontellaPereira et al. 1984). O. banksii subsp. banksii é encontrada principalmente na restinga, mas também foi coletada em mata secundária, pastos e beiras de estradas; ocorre apenas no território nacional, na Bahia e nos Estados do sul e sudeste (Fontella-Pereira et al. 1971). Na região de Viçosa, essas plantas foram encontradas em pastos e beiras de estradas. $\mathrm{O}$ presente estudo foi realizado na região de Viçosa (2045'S e 4251'W), Zona da Mata do Estado de Minas Gerais.

As polinizações manuais foram realizadas em indivíduos cultivados no Horto Botânico do
Departamento de Biologia Vegetal da Universidade Federal de Viçosa. Esses indivíduos foram conduzidos, cada um, inicialmente, em estaca de bambu e, posteriormente, sobre fios de nylon com aproximadamente $2,5 \mathrm{~m}$ compr. e ca. $1,2 \mathrm{~m}$ alt. do chão, amarrados em uma das extremidades no bambu ao início do desenvolvimento da planta e, na outra extremidade, em outra estaca de bambu; as plantas floresceram ao longo dos fios. Os tratamentos foram realizados com auxílio de lupa e o procedimento foi semelhante ao descrito por Kephart (1981). As flores (até 10 por indivíduo e sempre a primeira flor das inflorescências) foram isoladas na pré-antese com sacos de tecido do tipo organza. Na antese, foram etiquetadas e datadas. No terceiro dia após a antese (idade padrão de todas as flores utilizadas nas polinizações manuais), pela manhã, as flores foram polinizadas com polínias de flores também de três dias. Às autopolinizações foram realizadas com polínia da própria flor e as polinizações cruzadas foram realizadas entre plantas de locais diferentes.

As polinizações abertas foram realizadas em indivíduos de populações naturais. Para esse tratamento, foram etiquetadas inflorescências e suas flores permaneceram expostas aos visitantes florais. Para o cálculo da percentagem de frutificação, para cada espécie foi considerado o número médio de flores por inflorescência $(6,1$ para $O$. appendiculatum e 8,2 para $O$. banksii subsp. banksii; Vieira 1998).

As flores utilizadas para os cruzamentos interespecíficos também foram isoladas e, quando polinizadas, encontravam-se no terceiro dia de antese. Flores de $O$. appendiculatum receberam polínia de $O$. banksii subsp. banksii.

Os frutos resultantes dos testes supracitados, exceto os das polinizações abertas, permaneceram ensacados até a deiscência. Nessa ocasião, foram coletadas e contadas as sementes. Algumas ( 100 por espécie e dos híbridos $\mathrm{F}_{1}$ ) foram testadas quanto à percentagem de germinação, após serem colocadas em gerbox, sobre papel umedecido, e esses recipientes serem colocados em estufa com temperatura de $20-30^{\circ} \mathrm{C}$ e luz suplementar. As sementes consideradas viáveis foram as que apresentaram todas as 
estruturas (radícula, hipocótilo e plúmulas) normais, de acordo com as "Regras para análise de sementes" (Brasil 1992).

Algumas sementes de $F_{1}$ foram colocadas para germinar em sacos plásticos contendo terra e, quando apresentavam quatro ou cinco pares de folhas, foram transplantadas para canteiros experimentais no Horto Botânico. Nessas plantas foram realizadas autopolinizações e registrada a percentagem de flores férteis. Cem sementes de $\mathrm{F}_{2}$ também foram testadas quanto à percentagem de germinação.

\section{Resultados e discussão}

Polinização intra-específica - Os resultados das polinizações manuais em flores de $O$. appendiculatum e $O$. banksii subsp. banksii indicam que ambas são autocompatíveis, semelhantes a algumas espécies de Asclepias (Wyatt \& Broyles 1994; 1997), e que a viabilidade das sementes originadas das autopolinizações e das polinizações cruzadas é alta.

Os resultados das polinizações abertas indicam menor taxa de frutificação se comparada com as taxas obtidas nos tratamentos de polinizações manuais (Tab. 1), especialmente em $O$. banksii subsp. banksii. Vários autores têm considerado diversas hipóteses na tentativa de esclarecer a baixa taxa de frutificação em Asclepias (Willson \& Price 1980; Wyatt 1976; 1980; 1981; Bookman 1984; Queller 1985; Cabin et al. 1991). A produção de frutos nessas plantas é, segundo Wyatt (1981), o resultado de fatores extrínsecos, regulados pelo polinizador, e de fatores intrínsecos, regulados pela planta. Uma das hipóteses considerada, a incompatibilidade genética nos cruzamentos após autopolinizações (Bookman 1984; Wyatt \& Broyles 1990; fator intrínseco, Wyatt 1981), é inválida para as espécies aqui analisadas, pois ambas apresentam autocompatibilidade. A falta de polinizações (fator extrínseco, Wyatt 1981) parece ser o fator limitante da frutificação de $O$. banksii subsp. banksii, fato também observado em Asclepias speciosa (Bookman 1984), pois nos indivíduos cultivados a taxa de frutificação após polinizações manuais foi muito maior que a taxa obtida nas polinizações abertas. $\mathrm{O}$ mesmo parece não ocorrer em $O$. appendiculatum, na qual outros fatores, provavelmente intrínsecos, devem estar atuando (Tab. 1).

Polinização interespecífica - Os cruzamentos interespecíficos produziram sementes viáveis e os híbridos $O$. appendiculatum $\times O$. banksii subsp. banksii gerados indicam haver compatibilidade entre essas espécies. As autopolinizações realizadas em híbridos $F_{1}$ também produziram sementes viáveis (Tab. 2), indicando fertilidade nesses indivíduos. Entretanto, $36 \%$ das flores $(N$ = 564) apresentaram malformação dos polinários (unidade carregada pelos polinizadores e constituída de duas polínias e o translador; Bookman 1981), significando esterilidade masculina nessas flores.

Tabela 1. Resultados das polinizações manuais e polinizações abertas em flores de Oxypetalum appendiculatum e $O$. banksii subsp. banksii.

\begin{tabular}{|c|c|c|c|c|c|c|c|}
\hline \multirow{2}{*}{ Tratamento } & \multirow{2}{*}{$\begin{array}{c}\text { Indivíduos } \\
\left(\mathrm{n}^{\mathrm{g}}\right)\end{array}$} & \multirow{2}{*}{$\begin{array}{c}\text { Flores } \\
\left(n^{2}\right)\end{array}$} & \multicolumn{2}{|c|}{ Frutos $^{1}$} & \multicolumn{2}{|c|}{ Sementes/fruto } & \multirow{2}{*}{$\begin{array}{c}\text { Germinação } \\
\text { (\%) }\end{array}$} \\
\hline & & & $\left(\mathrm{n}^{\mathrm{2}}\right)$ & $(\%)$ & média & variação & \\
\hline & & \multicolumn{6}{|c|}{ Oxypetalum appendiculatum } \\
\hline Autopolinização & 2 & 43 & 10 & 23,2 & 143,8 & $113-161$ & 93,0 \\
\hline Polinização cruzada & 2 & 41 & 3 & 7,3 & 152,0 & $145-166$ & 97,0 \\
\hline Polinização aberta & 2 & 177 & 10 & 5,6 & - & - & - \\
\hline \multicolumn{8}{|c|}{ Oxypetalum banksii subsp. bamksii } \\
\hline Autopolinização & 2 & 17 & 11 & 64,7 & 148,1 & $86-282$ & 66,0 \\
\hline Polinização cruzada & 3 & 27 & 12 & 44,4 & 168,6 & $70-220$ & 82,0 \\
\hline Polinização aberta & 4 & 902 & 07 & 0,8 & - & - & - \\
\hline
\end{tabular}

'Fruto $=$ um ou dois folículos por flor 
Tabela 2. Resultados das polinizações interespecíficas entre Oxypetalum appendiculatum e $O$. banksii subsp. banksii e das autopolinizações do híbrido $\mathrm{F}_{\text {1. }}$.

Tratamento Indivíduos

Flores

Frutos ${ }^{1}$

Sementes/fruto

Germinação

(n')

(n)

(n⿳)

(\%)

média variação

Polinização interespecífica

\begin{tabular}{llllllll}
\hline O. appendiculatum & 6 & 20 & 7 & 35,0 & 113,9 & $76-149$ & 70,0 \\
O. banksii subsp. banksii & 1 & 11 & - & - & - & - & - \\
\hline \multicolumn{8}{c}{ Autopolinização do $F_{\text {, }}$} \\
\hline & 3 & 25 & 8 & 32,0 & 97,4 & $69-111$ & 95,0 \\
\hline
\end{tabular}

'Fruto = um folículo por flor

$\mathrm{O}$ híbrido $O$. appendiculatum x $O$. banksii subsp. banksii não foi encontrado na natureza (Fontella-Pereira, com. pes.) o que significa que as barreiras de isolamento reprodutivo entre as espécies parentais não foram quebradas. Se houver a quebra, a direção mais provável do cruzamento seria $O$. appendiculatum exercendo a função feminina, como realizado no presente trabalho, devido às características florais das plantas estudadas (Vieira 1998); isolamento mecânico unilateral foi observado por Kephart \& Heiser (1980) em espécies de Asclepias.

\section{Agradecimentos}

À CAPES, pela concessão de Bolsa de Doutorado à primeira Autora.

\section{Referências bibliográficas}

Barroso, G. M.; Guimarães, E. F.; Ichaso, C. L. F.; Costa, C. G.; Peixoto, A. L. \& Lima, H. C. 1986. Sistemática de Angiospermas do Brasil. vol 3. Imprensa Universitária, Viçosa.

Bookman, S. S. 1981. The floral morphology of Asclepias speciosa (Asclepiadaceae) in relation to pollination and a clarification in terminology for the genus. American Journal of Botany 68: 675-679.

Bookman, S. S. 1984. Evidence for selective fruit production in Asclepias. Evolution 38: 72-86.

Brasil, Ministério da Agricultura e Reforma Agrária. 1992. Regras para análise de sementes. Brasília.

Cabin, R. J.; Ramstetter, J. \& Engel, R. E. 1991. Reproductive limitations of a locally rare Asclepias. Rhodora 93: 110.

Fontella-Pereira, J.; Valente, M. da C. \& Alencastro, F. M. M. R. de. 1971. Contribuição ao estudo das Asclepiadaceae brasileiras. V. Estudo taxonômico e anatômico de
Oxypetalum banksii Roem. et Schult. Rodriguésia 38: 261-281.

Fontella-Pereira, J.; Valente, M. da C. \& Schwarz, E. de A. 1984. Contribuição ao estudo das Asclepiadaceae brasileiras. XXI. Asclepiadaceae do município de Ouro Preto, Estado de Minas Gerais - Uma sinopse. Boletim do Museu Botânico Kuhlmann 2: 63-127.

Kephart, S. R. 1981. Breeding systems in Asclepias incarnata L., A. syriaca L., and A. verticillata L. American Journal of Botany 68: 226-232.

Kephart, S. R. \& Heiser, C. B. 1980. Reproductive isolation in Asclepias: lock and key hypothesis reconsidered. Evolution 34: 738-746.

Kephart, S. R.; Wyatt, R. \& Parrella, D. 1988. Hybridization on North American Asclepias. I. Morphological evidence. Systematic Botany 13: 456473.

Mabberley, D. J. 1997. The plant-book - a portable dictionary of the vascular plants. University Press, Cambridge.

Occhioni, P. 1953. Notas sôbre o gênero Oxypetalum - II (As espécies do Estado do Rio de Janeiro). Dusenia 4: 251-271.

Ollerton, J. \& Liede, S. 1996. Fly pollination in the Asclepiadaceae: old data and new techniques Pp. 10. In: Reproductive biology 96, Systematics, conservation and economic botany. Abstracts.

Queller, D. C. 1985. Proximate and ultimate causes of low fruit production in Asclepias exaltata. Oikos 44: 373-381.

Rosatti, T. J. 1989. The genera of suborder Apocynineae (Apocynaceae and Asclepiadaceae) in the southeastern United States. Journal of the Arnold Arboretum 70: 443-514.

Sparrow, F. K. \& Pearson, N. L. 1948. Pollen compatibility in Asclepias syriaca. Journal of Agricultural Research 77: 187-199.

Stevens, O. A. 1945. Asclepias syriaca and A. speciosa, distribution and mass collections in North Dakota. The American Midland Naturalist 34: 368-374.

Swarupanandan, K.; Mangaly, J. K.; Sonny, T. K.; Kishorekumar, K. \& Chand-Basha, S. 1996. The subfamilial and tribal classification of the family Asclepiadaceae. Botanical Journal of the Linnean Society 120: $327-369$. 
Vieira, M. F. 1998. Biologia reprodutiva de espécies de Oxypetalum (Asclepiadaceae), na região de Viçosa, MG, sudeste brasileiro. Tese de Doutorado. UNICAMP, Campinas.

Willson, M. F. \& Price, P. W. 1980. Resource limitation of fruit and seed production in some Asclepias species. Canadian Journal of Botany 58: 2229-2233.

Woodson, R. E. 1954. The North American species of Asclepias L. Annlas of the Missouri Botanical Garden 41: 1-211.

Wyatt, R. 1976. Pollination and fruit-set in Asclepias: a reappraisal. American Journal of Botany 63: 845851 .

Wyatt, R. 1980. The reproductive biology of Asclepias tuberosa: I. Flower number, arrangement, and fruit-set. New Phytologist 85: 119-131.
Wyatt, R. 1981. The reproductive biology of Asclepias tuberosa: II. Factors determining fruit-set. New Phytologist 88: 375-385.

Wyatt, R. \& Broyles, S. B. 1990. Reproductive biology of milkweeds (Asclepias): recent advances. In: S. Kawano (Ed.), Biological Approaches and Evolutionary Trends in Plants. Academic Press, London.

Wyatt, R. \& Broyles, S. B. 1994. Ecology and evolution of reproduction in milkweeds. Annual Review of Ecology and Systematics 25: 423-441.

Wyatt, R. \& Broyles, S. B. 1997. The weedy tropical milkweeds Asclepias curassavica and A. fruticosa are self-compatible. Biotropica 29: 232-234.

Wyatt, R. \& Hunt, D. M. 1991. Hybridization in North American Asclepias. II. Flavonoid evidence. Systematic Botany 16: 132-142. 